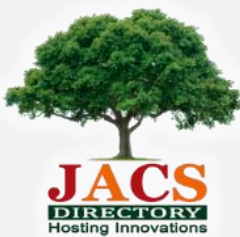

\title{
Studies on Electrical and Optical Property of Polyaniline/ZnO Nanocomposites
}

\author{
Bharati Basavaraj*, Basavaraja Sannakki \\ Department of Post Graduate Studies and Research in Physics, Gulbarga University, Kalaburagi - 585 106, Karnataka, India.
}

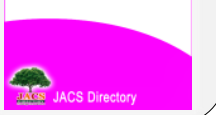

\section{ART T C LEDETAILS}

\section{Article history:}

Received 14 December 2020

Accepted 29 December 2020

Available online 17 January 2021

\section{Keywords:}

Polymerisation

Nanocomposite

ZnONPs

\begin{abstract}
A B S T R A C T
The conducting polymer polyaniline and PANI/ZnO nanocomposites were synthesized by in-situ polymerization method at $0-5^{\circ} \mathrm{C}$ temperature with ammonium persulphate as an oxidising agent. The optical band gap of PANI/ZnO nanocomposites have been studied at different weight percentage such as $10 \%, 30 \%$ and $50 \%$, at room temperature. The XRD pattern confirmed the hexagonal wurtzite structure of the $\mathrm{ZnO}$ product. The particle size was calculated by using the Debye-Scherrer relation and it was found to be in the range of $20-50 \mathrm{~nm}$. The obtained absorption spectra at $385 \mathrm{~nm}$ is the charectestic peak of $\mathrm{ZnO}$ and observed for all weight percentages of nanocomposites. The peaks occured at $300 \mathrm{~nm}$ and $470 \mathrm{~nm}$ confirms the formation of polyaniline in the nanocomposites. Further the dielectric properties such as dielectric constant, dielectric loss and AC conductivity for the PANI with PANI/ZnO composites at different weight percentages are studied as a function of frequency in the range $5 \mathrm{~Hz}-35 \mathrm{MHz}$ at room temperature.
\end{abstract}

\section{Introduction}

Polymer nanocomposites in recent years have become one of the most extensively studied materials all over the world due to their unusual properties of the synthesis of conducting polymers like polyaniline (PANI), poly-pyrrole (PPy) and polythiopene (PTh) due to their high electrical conductivity, interesting electrochemical properties, and easy processability [1-3]. These electrical and electro-chemical properties of such conducting polymers show much assurance for commercial applications in battery materials, electrochromic devices, sensor technology and nonlinear optics (NLO) [4]. Recent years several applications for the control of band gap is essential for light emitting diodes (LED) transparency in the visible region combined with high electrical conductivity etc., [5]. However, among various aromatic compound based conducting polymers, polyaniline is one of the most attractive conducting polymers due to its special transport properties, facile synthesis, tunable conductivity and good environmental stability [6]. Hence nanocrystalline materials are considerable interested due to their unique dielectric, magnetic and optical properties.We have selected zinc oxide nanoparticles due to their importance in wide range of applications in lithium batteries, high-density information storage devices, radar absorbing materials, magnetic fluids [7]. The semiconductor $\mathrm{ZnO}$ is a conventionaly having wide band-gap. Its unique properties make it appropriate for various biomedical applications, such as anticancer, antibacterial, and antifungal uses [8-10]. The semiconductor properties of $\mathrm{ZnO}$ affect their ability to generate reactive oxygen species. The electrons in semiconductors contain energies within certain bands and the band gap, crystalline $\mathrm{ZnO}$ measures $3.3 \mathrm{eV}$ for a void region extending from top of the filled valence band to the bottom of vacant conduction band. UV light contain sufficient energy to promote electron to the conduction band, leaving behind holes $(\mathrm{h}+)$. The electron and hole migrate to the surface of the nanoparticles and react with oxygen and hydroxyl ions, respectively. This leads to the formation of superoxide and hydroxyl radicals [11]. In this work we report optical absorbance, band gap, of pure polyaniline and polyaniline doped with zinc oxide $(\mathrm{ZnO})$ nanoparticles.

\section{Experimental Methods}

\subsection{Materials}

Analytical reagent grade (AR) monomer pyrrole, oxidising agent ammonium persulphate (APS), zinc nitrate hexa hydrate, acetone and $\mathrm{HCl}$, Whatman filter papers, double distilledwater were used for the synthesis of polyaniline and Aloe vera plant used for green synthesis of $\mathrm{ZnO}$ nanoparticles.

\subsection{Green Synthesis of ZnO Nanoparticles}

The zinc oxide nanoparticles were synthesized by 'self-propagating low temperature combustion method' as shown in Fig. 1, employing zinc nitrate hexahydrate $\left(\mathrm{Zn}\left(\mathrm{NO}_{3}\right)_{2} \cdot 6 \mathrm{H}_{2} \mathrm{O}\right)$ as a precursor and Aloe vera gel as a fuel.

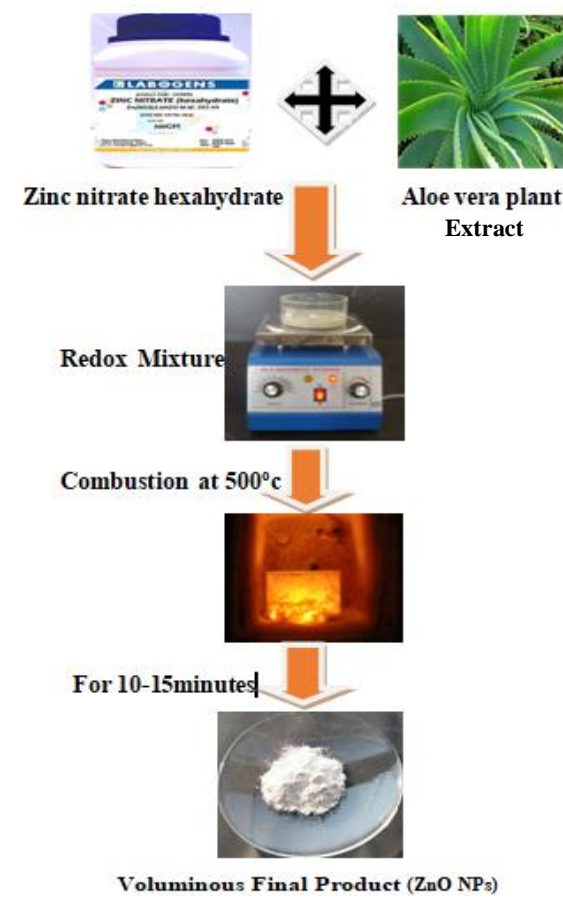

Fig. 1 Flow chart for the synthesis of $\mathrm{ZnO}$ nanoparticles 
A $10 \mathrm{~mL}$ of Aloe vera leaf extracted gel solution was prepared and then $2.14 \mathrm{~g}$ of zinc nitrate was added with the extract and kept on a magnetic stirrer for $10 \mathrm{~min}$ constant stirring to get homogeneous solution. The uniform mixture of both oxidizer as well as the fuel was introduced into the pre-heated muffle furnace kept at $450^{\circ} \mathrm{C}$. The mixture boils with froth finally yielded a white powder of $\mathrm{ZnO}$ nanoparticles.

\subsection{Synthesis of Polyaniline and PANI/ZnO Nanocomposites}

The monomer aniline and oxidising agent ammonium persulphate (APS) used as catalyst were of analytical grade and procured from SD Fine Chemicals, India. The pyrrole monomer was used as received for the synthesis of polyaniline without further purification. The polymeric materials were prepared via chemical oxidative techcnique using APS as an oxidant. In the typical procedure, monomer and oxidant in stiochometric ratio 1:1 were dissolved in deionized water. Consequently, $\mathrm{HCl}(0.3 \mathrm{M})$ was added into the reaction mixture results aniline hydrochloride. The reaction mixture was allowed for constant stirring to six hour on a magnetic stirrer at $5{ }^{\circ} \mathrm{C}$. Then it was kept for overnight at room temperature for complete polymerization reaction. The prepared solution is filtered using Whatman No. 41 filter paper. Then the obtained precipitate was washed with copious amounts of acetone and double distilled water until to get colorless filtrate. Subsequently to this step, sample was sintered at $60^{\circ} \mathrm{C}$ for 12 hours then it is dried completely which is to be grinded with the help of agate mortar. The same procedure repeated in thrice. In the same manner three samples were prepared at different weight percentge such as $10 \%, 30 \%$ and $50 \%$. The same samples were used to carried out charectarizations such as UV-visible spectroscopy, XRD and dielectric properties such as dielectric constant, dielectric loss and AC conductivity for the PANI with PANI/ZnO composites at different weight percentages as a function of frequency in the range $5 \mathrm{~Hz}-35 \mathrm{MHz}$ at room temperature.

\section{Results and Discussion}

\subsection{UV-Vis Spectrophotometer}

The optical properties of the polyaniline and nanocomposites were studied using a double-beam monochromatic UV-Vis spectrophotometer. The optical absorption data of the polymer samples were recorded in the range of $200 \mathrm{~nm}$ to $800 \mathrm{~nm}$ at room temprature by using T90+ UV-visible spectrophotometer. UV-vis absorption spectra of polyaniline and PANI/ZnO nanocomposites are shown in Fig. 2. The spectrum of synthesized material exhibits absorption around the $335-470 \mathrm{~nm}$ which is the distinctive peak of polyaniline. The two different absorption peaks, at $335 \mathrm{~nm}$ and $470 \mathrm{~nm}$ were due to wide chain length distribution of polymer [12]. polyaniline exhibits two bands at $335 \mathrm{~nm}$ corresponding to $\pi \rightarrow \pi^{*}$ inter band transition and the other band at $470 \mathrm{~nm}$ which is also corerspounds to polaron band transitions, whereas the small peak at 385 $\mathrm{nm}$ was observed for doped polyaniline with $\mathrm{ZnO}$ nanoparticles which is attributed to transitions of the valence to polaron and bipolaron states which is the characteristic peak of $\mathrm{ZnO}$ nanoparticles $[13,14]$. The peak shift in wavelengths are observed after doping. The wavelength is shifted from $335 \mathrm{~nm}$ to $338 \mathrm{~nm}$ for nanocomposites of $10 \%$ and $30 \%$; for $50 \%$, the wavelength is shifted from $335 \mathrm{~nm}$ to $340 \mathrm{~nm}$.

The optical band gap can be measured from its UV-visible absorption spectra. The absorption coefficient $(\alpha)$ was calculated using the following Eq.(1) [15],

$$
\alpha(v)=2.303 \frac{A}{t}
$$

where $A$ is the optical absorbance and $t$ is the thicknessof the sample. Here the sample is taken as solution, hence thickness is equal to cuvette length (sample cell) size as $1 \mathrm{~cm}$. The value and nature of the optical band gap depends on absorption coefficient.The optical band gap can be calculated using the following Eq.(2) [16].

$$
(\alpha h v)^{\mathrm{n}}=\mathrm{B}\left(\mathrm{hu}-\mathrm{E}_{\mathrm{g}}\right)
$$

where $\alpha$ is absorption coefficient, h is Planck's constant, $v$ is the frequency, $\mathrm{B}$ is a constant which depends on the transition probability, $\mathrm{E}_{\mathrm{g}}$ is the optical band gap and $\mathrm{n}$ is an index which indicates the optical absorption process. Theoretically, it is equal to 2 for direct allowed transition, $1 / 2$ for indirect allowed transition, $2 / 3$ for direct forbidden transition and $1 / 3$ for indirect forbidden transition [17]. In the present case, the band gap has been calculated for pure polyaniline and PANI/ZnO nanocomposite at $50 \%$. For the determination of optical band gap, $(\alpha h v)^{2}$ was plotted as a function of photon energy $h v$. The value of direct band gap $\left(E_{g}\right)$ was calculated from the intersection of the extrapolated line with the photon https://doi.org/10.30799/jnst.318.21070102 energy axis $(\alpha h v)^{2}=0$ using the linear portion of absorption edge of the UVVis absorption spectra. By the calculation, the optical band gap for pure polyaniline and PANI/ZnO nanocomposites are obtained as $3.76 \mathrm{eV}$ and $3.68 \mathrm{eV}$ respectively.

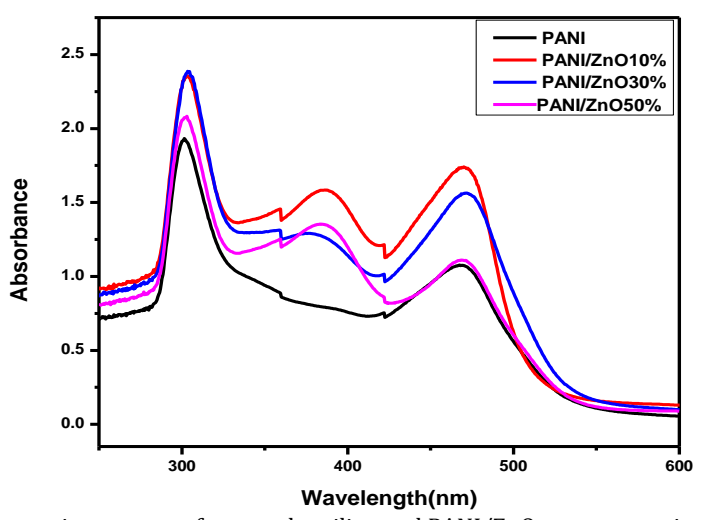

Fig. 2 Absorption spectra of pure polyaniline and PANI/ZnO nanocomposites

\subsection{X-Ray Diffraction Analysis}

To study the nature of crystallinity or amorphous, powder XRD characterisation have been done for pure PANI, pure PVDF and polymer blended PANI with PVDF/KDP composites. The X-ray diffraction patterns of the samples are obtained using a Philips X-ray diffractometer with $\mathrm{CuK \alpha}$ radiation $(\lambda=1.54060 \AA)$. The diffractograms were recorded in terms of $2 \theta$ in the range 10 to 90 degrees with a scanning rate of 4 degrees per minute. Figs. 3-5 show the XRD pattern of synthesized pure PANI, ZnO NPs and polymer PANI/ZnO composites. PANI X-ray diffraction pattern at Fig. 3 shows a broad peak occurred at $2 \theta=25.58^{\circ}$ which is the characteristic peak says the amorphous nature of PANI [18].

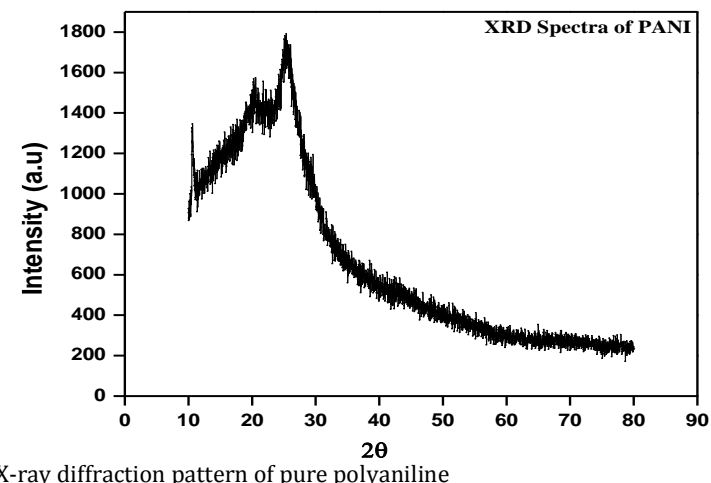

Fig. 3 X-ray diffraction pattern of pure polyaniline

\subsection{XRD Analysis of ZnO Nanoparticles}

It is observed from Fig. 4 that the powder XRD patterns of ZnO NPs characteristic diffraction peaks of $\mathrm{ZnO}$ including the planes (100), (002), (101), (102), (110), (103), (200), and (112), (201), (004), (202) were observed at an angle $31^{\circ}, 34^{\circ}, 36^{\circ}, 47^{\circ}, 56^{\circ}, 63^{\circ}, 66^{\circ}, 67^{\circ}, 69^{\circ}, 72^{\circ}$, and $77^{\circ}$ for the synthesised $\mathrm{ZnO}$ nanoparticle sample. All the diffraction peaks are well assigned to the hexagonal wurtzite phase of ZnO (JCPDS card No. 361451) [19]. The presence of the high intensity peaks inferred that the prepared sample were highly crystalline in nature. The average particle size of the $\mathrm{ZnO}$ nanoparticles was found by using Debye-Scherer formula is $20-50 \mathrm{~nm}$.

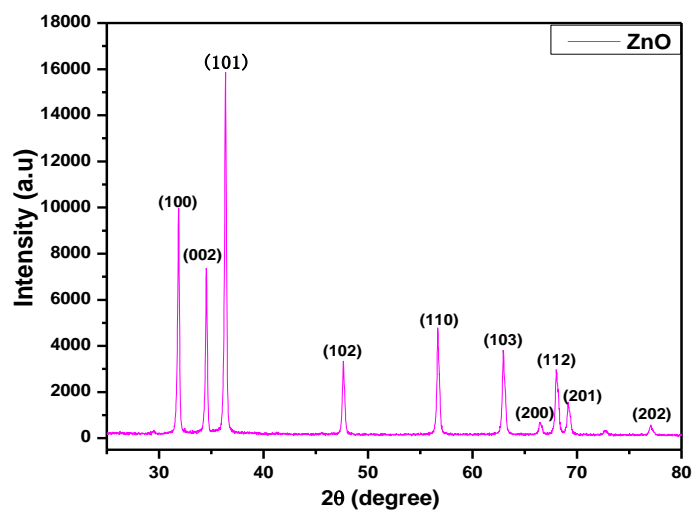

Fig. 4 X-ray diffraction pattern of $\mathrm{ZnO}$ nanoparticles 


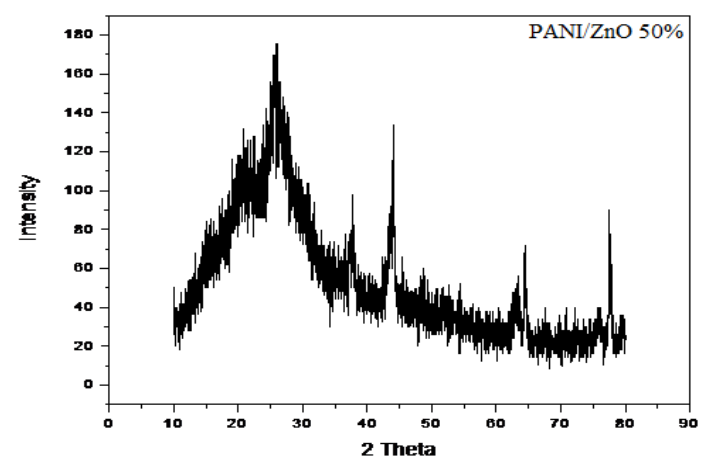

Fig. 5 The X-ray diffraction pattern for PANI and PANI/ZnO composites

Fig. 5 shows the X-ray diffraction pattern of PANI with ZnO polymer nanocomposites and as wt.\% of ZnO NPs increases in polymer matrix the intensity of PANI increases which indicate the semi crystalline nature of prepared sample PANI/ZnO composites [20,21].

\subsection{Dielectric Properties}

The dielectric properties have been studied for all the PANI and PANI/ZnO composites as a function of frequency at room temperature.

\subsubsection{Dielectric Constant}

The dielectric constant with frequency for PANI and PANI/ZnO composites of different weight percentages are shown in the Fig. 6. Using the values of capacitance the dielectric constant were determined for PANI and PANI/ZnO at 10, 30, and $50 \mathrm{wt} \%$, using the expression Eq.(3) [22]

$$
\varepsilon^{\prime}=\frac{C d}{\varepsilon_{0} A}
$$

where $\mathrm{d}$ is the thickness of the sample, $\mathrm{A}$ is the effective cross-sectional area of the sample, $\mathrm{C}$ is the capacitance of the sample. The dielectric constant of PANI and PANI/ZnO composites decreased as frequency increased up to $180.9 \mathrm{~Hz}$, which is shown in Fig. 6. The dielectric constant remains almost constant after frequency of $180.9 \mathrm{~Hz}$ and it shows independent of frequency because of electrical relaxation process. It is observed that for PANI and PANI/ZnO composites as weight percentage of ZnO NPs increases the dielectric constant decreases. The dielectric constant decreases due to the shorter time available for the dipoles to align [23].

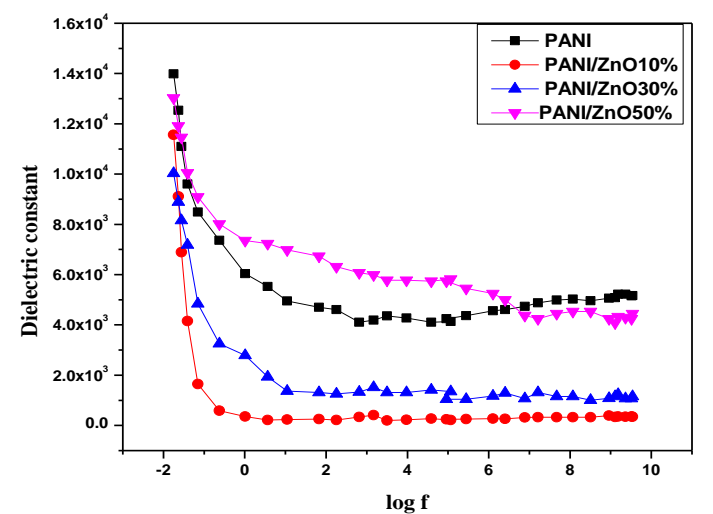

Fig. 6 Dielectric constant with frequency for PANI and PANI/ZnO composites

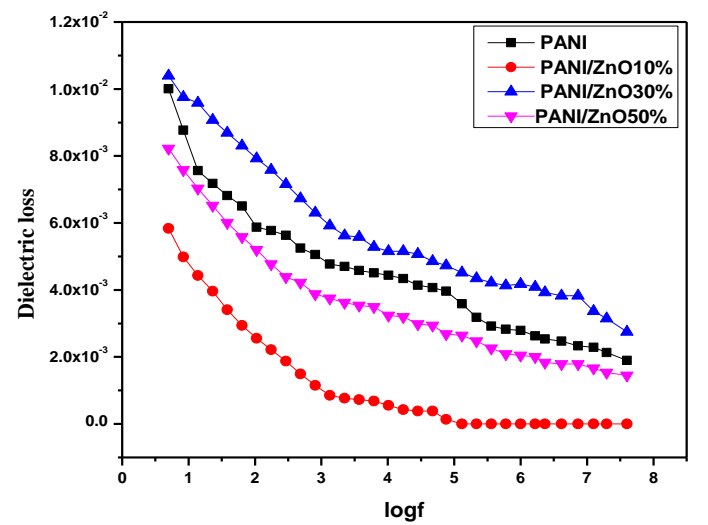

Fig. 7 The graph of dielectric loss with frequency for PANI and PANI/ZnO composites https://doi.org/10.30799/jnst.318.21070102

\subsubsection{Dielectric Loss}

The dielectric loss with frequency for PANI and PANI/ZnO composites of different weight percentages are shown in the Fig. 7. The dielectric loss is obtained with the help of experiment data of dissipation factor and the values of dielectric constant using the relation given by,

$$
\varepsilon^{\prime \prime}=\varepsilon^{\prime} \tan \delta
$$

Fig. 7 shows dielectric loss decreased gradually as frequency increased higher values for all the composites of PANI/ZnO. But for PANI it decreases as the frequency increases for higher values. The dielectric loss $\varepsilon "$ decreases due to the migration of ions in the material [24].

\subsubsection{AC Conductivity}

The AC conductivity as a function of frequency for PANI and PANI/ZnO composites of different weight percentages at room temperature are shown in the Fig. 8

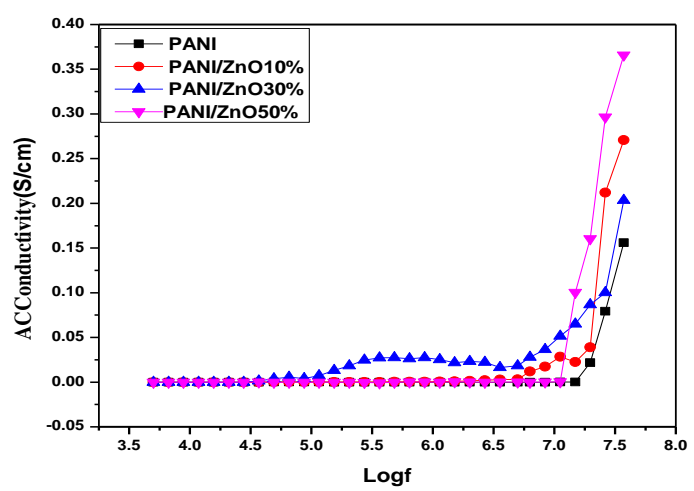

Fig. 8 AC conductivity with frequency for PANI and PANI/ZnO composites

The electrical property of $\mathrm{AC}$ conductivity $\left(\sigma_{\mathrm{ac}}\right)$ as a function of frequency have been determined by dielectric data using the following equation,

$$
\sigma \mathrm{ac}=\varepsilon^{\prime} \varepsilon_{\mathrm{o}} \omega \tan \delta
$$

where $\varepsilon_{\mathrm{o}}$ is the permittivity of free space $=8.85 \times 10^{-12} \mathrm{Fm}^{-1}, \omega$ is the angular frequency and $\varepsilon^{\prime}$ is the dielectric constant. From Fig. 8, it is observed that in all the cases, $\sigma_{\mathrm{ac}}$ (AC conductivity) remains constant up to $7.5 \mathrm{MHz}$ for PANI and PANI/ZnO composites. Afterwards it is increased for higher frequencies. As weight percentage of $\mathrm{ZnO}$ increases in polyaniline matrix the AC conductivity increases due to interfacial polarization. It is observed from Fig. 8 for $50 w t \%$ of polymer nanocomposites is high [24]. But for 10 and $30 \mathrm{wt} \%$ of PANI/ZnO composites the AC conductivity is decreased because of dipole polarization.

\section{Conclusion}

The present work confirms the $\mathrm{ZnO}$ nanoparticles were synthesized by green combustion technique using Aloe vera leaf extract as a fuel. The Xray diffraction pattern of ZnO NPs confirms crystaline nature. It was also found that the particle size in the range $20-50 \mathrm{~nm}$, calculated by DebyeScherrer equation. It was good agreement with JCPDS card No. 36-1451. The dielectric properties of PANI/ZnO composites' various wt.\% were studied in the frequency range from $5 \mathrm{~Hz}-35 \mathrm{MHz}$. Dielectric measurement indicates that the prepared samples has great influence on the dielectric behavior due to $\mathrm{ZnO}$ nanoparticles. From the optical properties studies absorption of sample using T90+UV-vis spectrophotometer the characteristic peaks are observed at $335 \mathrm{~nm}$ and $470 \mathrm{~nm}$ and it reveals that the presence of polyaniline in polymer nanocomposites. Also the band gap of pure PANI was found to be $3.76 \mathrm{eV}$ and $3.68 \mathrm{eV}$ for $\mathrm{ZnO}$ NPs. Due to these wide band gap there may be useful applications in the field of electric optoelectronic devices.

\section{Acknowledgement}

The authors are grateful to the Chairpersons, Department of Physics and USIC Department at GUK, for providing necessary characterization facilities.

\section{References}

[1] S.J. Pearton, D.P. Norton , K. Ip, Y.W. Heoa, T. Steiner, Recent advances in processing of ZnO, J. Vac. Sci. Technol. 50 (2005) 293-340. 
[2] D.C. Look, J.W. Hemsky, J.R. Sizelove, Residual native shallow donor in ZnO, Phys. Rev. Lett. 82 (1999) 2552-2555.

[3] C.G. Van de Walle, Hydrogen as a cause of doping in zinc oxide, Phys. Rev. Lett. 85 (2000) 1012-1015.

[4] A. Janotti, C.G. Van de Walle, Fundamentals of zinc oxide as a semiconductor, Rep. Prog. Phys. 72 (2009) 126501:1-29.

[5] D.C. Look, Recent advances in ZnO materials and devices, Mater. Sci. Eng. B 80 (2001) 383-387.

[6] C. Jagadish, S.J. Pearton, Zinc oxide bulk, thin films and nanostructures: processing, properties, and applications, Elsevier, New York, 2006.

[7] O. Schmidt, P. Kiesel, C.G. Van de Walle, N.M. Johnson, J. Nause, G.H. Dohler, Effects of an electrically conducting layer at the zinc oxide surface, Japan. J. Appl. Phys. Part 1, 44 (2005) 7271-7274.

[8] D. Fernandez-Hevia, M. Peiteado, J. De Frutos, A.C. Caballero, J.F. Fernandez, Wide range dielectric spectroscopy of $\mathrm{ZnO}$-based varistors as a function of sintering time, J. Eur. Ceram. Soc. 24 (2004) 1205-1208.

[9] Zhimin Dang, Lizhen Fan, Shujin Zhao, Cewen Nan, Dielectric properties and morphologies of composites filled with whisker and nanosized zinc oxide, Mater. Res. Bull. 38 (2003) 499-507.

[10] M. Soosen Samuel, Jiji Koshy, Anoop Chandran, K.C. George, Electrical charge transport and dielectric response in ZnO nanotubes, Curr. Appl. Phys. 11 (2011) 1094-1099.

[11] J.C. Maxwell, A treatise on electricity and magnetism, Vol. 1, Oxford University Press, Oxford, England, 1873.

[12] L.Joshi, R.Prakash. One-pot synthesis of polyindole-Aunanocomposite and its nanoscale electrical properties, Mat. Let. 65 (2011) 3016-3019.

[13] S. Krishnaswamya, V. Ragupathia, S. Ramana, P. Panigrahia, G.S. Nagarajan, Study of optical and electrical property of NaI-doped PPy thin film with excellent photocatalytic property at visible, Polym. Bull. 76 (2019) 5213-5231.
[14] K. Ponprapakaran, R. Harihara Subramani, R. Baskaran, R. Anbarasan, Synthesis, characterization, and application of fluorescent electrically conducting copolymer/metal-oxide nanocomposites, Synth. Met. 232 (2017) 144-151.

[15] M. Fox, Optical properties of solids Oxford University Press, New York, 2001.

[16] F.A. Mustafa, Optical properties of NaI doped polyvinyl alcohol films, Phys. Sci. Res. Int. 1 (2013) 1-9.

[17] A.P. Indolia, M.S. Gaur, Optical properties of solution grown PVDF-ZnO nanocomposite thin films, J. Polym. Res. 20(1) (2013) 1-8.

[18] A.Hezam,K. Namratha, Q.A. Drmosh, B.N.Chandrashekar, K.K. Sadasivuni, et al., Heterogeneous growth mechanism of $\mathrm{ZnO}$ nanostructures and the effects of their morphology on optical and photocatalytic properties, Cryst. Eng. Comm. 19(24) (2017) 3299-3312.

[19] G. Murugadoss, Synthesis and characterization of transition metals doped ZnO nanorods, J. Mater. Sci. Technol. 28(7) (2012) 587-593.

[20] D. Zhang, J. Zhang, Z. Guo, X. Miao, Optical and electrical properties of zinc oxide thin films with low resistivity via Li-Ndual-acceptor doping, J. Alloy. Comp. 509(20) (2011) 5962-5968.

[21] S. Mandal, K. Sambasivarao, A. Dhar, S.K. Ray, Photoluminescence and electrical transport characteristics of $\mathrm{ZnO}$ nanorods grown by vapor-solid technique, J. Appl. Phys. 106 (2009) 024103:1-8.

[22] Sannakki Nagaraja, S.M. Ambalagi, H.K. Inamdar, B. Bharathi, D. Mahalesh, et al., Synthesis and characterization of PANI/PVDF composites for dielectric and AC conductivity, J. Nanosci. Tech. 4(5) (2018) 516-518.

[23] M.H. Abdullah, A.N. Yusoff, Frequency dependence of the complex impedances and dielectric behaviour of some Mg-Zn ferrites, J. Mater. Sci. 32 (1997) 5817 5823.

[24] A.K. Jonscher, The universal dielectric response, Nature 267 (1977) 673-679. 